\title{
A Case of Personalized Management of Carcinoma of Fallopian Tube Harboring BRCA Mutation and Sister Mary Joseph's Nodule
}

\section{Xi Chen}

Weifang Medical University

\section{Yaoyao Fu}

Weifang Medical University

\section{Na Zhang}

Affiliated Hospital of Weifang Medical University

\section{Yang Li}

Weifang Medical University - Fuyanshan Campus: Weifang Medical University

Min Zhang

Weifang Medical University

guili wang ( $\nabla$ guiliwang78@163.com )

Affiliated Hospital of Weifang Medical University

\section{Research Article}

Keywords: BRCA, PGD/PGS, Primary high-grade serous Carcinoma of Fallopian Tube, Sister Mary Joseph's nodule

Posted Date: May 17th, 2021

DOl: https://doi.org/10.21203/rs.3.rs-386598/v1

License: (c) (1) This work is licensed under a Creative Commons Attribution 4.0 International License. Read Full License 


\section{Abstract}

Fallopian tube malignancies have been presumed to rare incidence with dismal prognosis. However, recent data suggest that the true incidence of PFTC has been substantially underestimated. Early diagnosis of fallopian tube cancer seems difficult. The routes of metastasis of fallopian tube carcinoma are similar to those of ovarian cancer. Genetic factors are relatively important risk factors for tubal cancer. Poly ADP -ribose polymerase inhibitor is used for BRCA-mutant PFTC. In this artical, we use a rare case of navel metastatic tumor which finally be confirmed that it is originated from fallopian tube to discovered the hereditary factors through genetic testing, and reported this case to provide preventive intervention for the next generation of eugenics and incidence.

\section{Precis}

A fallopian tube carcinoma female with Sister Mary Joseph's nodule and positive BRCA2, we reported this case to provide preventive intervention for the next generation of eugenics and incidence.

\section{Introduction}

Fallopian tube malignancies have been presumed to rare incidence with dismal prognosis. However, recently, histology and molecular genetics give strong evidence that up to $80 \%$ oftumor that were classified as high-grade serous carcinomas of the ovaries and peritoneum may have originated in the fimbrial end of the fallopian tube. Reasonably, gynecologic Oncology Committee of FIGO revised the staging to incorporate ovarian, fallopian tube, and peritoneal cancer in the same system in $2014^{1}$. The treatment for fallopian tube malignancies is in accordance with ovarian cancer guidelines. Pelvic mass, watery or blood discharge and abdominal pain are called a triad of primary fallopian tube carcinoma ${ }^{2}$. But coincidence are rare. In fact, very few data exist on the treatment. difficulties remain in diagnose and treatment due to its late detection and familial heredity character. The same to ovary cancer, the strongest risk factor of primary fallopian tube carcinoma is the famiily history of breast or ovarian cancer. Approximately $25 \%$ of all ovarian cancers are caused by a heritable genetic condition. Of these, mutations in BRCA1 and BRCA2 account for almost $40 \%$ of ovarian cancer cases in women with a family history of the disease ${ }^{3}$. Increased awareness of potential molecule change of the disease avail to personalized diagnosis, prediction and remedy and bring about clinical significance for her family members. Up to now, it's available to receive genetic testing to screen the virulence gene. As for the women with positive screening result, it's likely to pass the gene to her next generation. Thus, someone with high risk factor should pay more attention to it. In consideration of its difficulty in treatment and high mortality. We should focus more on prevention. But how to avoid it? Preimplantation genetic diagnosis/screening(PGD/PGS) is a good choice. By screening high-quality embryos without genetic mutations for transplantation. What's more, as a route of the tumor metastasis, Umbilical metastasis(Sister Mary Joseph's nodule) is fairly rare ${ }^{4}$. fallopian tube cancer is no exception. Here we 
describe a fallopian tube carcinoma female with Sister Mary Joseph's nodule and positive BRCA2 as follows.

\section{Case Report}

A 57-year-old, Chinese, married female, with gravida 7, para 2, abortus 4, living children 2, ectopic pregnancy 1, (G7P2A4L2Ep1), natural menopause at 55-yr-old, complained of "swelling and ache in umbilicus, generating pus after repture for 2 months", experienced operations twice a month in May 2020 . Macroscopically, in the middle of the navel, there is rupture lesion, surrounded by hyperplasia of granulation tissue, with a small amount of yellow-white pus outflow after extrusion, bringing about a lesion measuring $2.5 \times 2 \times 1.5 \mathrm{~cm}$. Histopathologic examination of the tissue revealed glandular, papillary adenocarcinoma tissue under squamous epithelium, with multitudinous sand bodies.

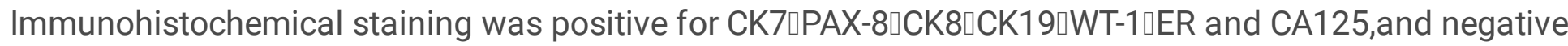

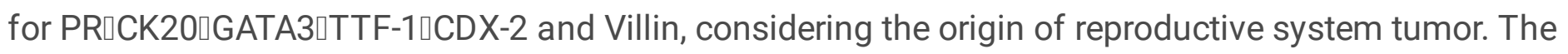
above-mentioned findings overturned what we diagnosed omphalitis. But in fact, it's consistent with Sister Mary Joseph's nodule.

In the review of the patient's past history, she had hypertension and diabetes for about 2 years and an right salpingectomy 27 years ago. Her menstrual and childbearing history revealed that 12 5-7/28-30. She gave birth to two sons naturally. She had a family history of breast cancer $\square$ ovarian cancer and diabetes. The physical examination gynecological examination seems nothing special. None of palpable masses and cysts in the breast labdominal masses around the whole abdomen and adnexal masses in the pelvic cavity. After the first surgery, in the view of Sister Mary Joseph's nodule, the serum tumor markers began to be tested, it showed : CA 125: 47. 23U/ml, CA $15-3: 12.08 \mathrm{U} / \mathrm{ml}$; CA $19-9: 69$. 40U/ml; HE 4 : 71. 88pmol/l; POSTROMA: $30.53 \%$. Computed tomography (CT) scanning of the chest displayed: localized bronchiectasis in the lower lobe of the right lung with a little inflammation in the surrounding lung; liver abscesses were possible; Mixed density shadows near the liver margin at the top of the liver. CT of the whole abdomen and pelvis demonstrated multiple cystic lesions at the top of the liver with partial calcification, high fat density around the umbilicus, and uterus with multiple calcifications. Further examination, magnetic resonance imaging of upper abdomen and pelvis displayed : many abnormal signals at the top of the liver, some DWIBS signals slightly enriched; postoperative change in peri-umbilicus, some DWIBS signals enriched; slightly enlarged lymph nodes in retroperitoneal, pelvic and bilateral inguinal area; nodule between uterine body and cervix, considering myoma of uterus; liver, peri-umbilicus, uterus lesion, dynamic enhancement of the whole abdomen recommended; HPV and TCT revealed no abnormalities. She was discharged because of personal reason soon. She had an out-ofhospital consultation indicating serous carcinoma, suggesting further inspection to uterus and two-sided adnexa.

For this reason, the patient were readmitted to ask for further treatment. After prooperative discussion, she experienced a Laparoscopic exploration. During laparoscope, it's visible to a small amount of pale yellow cloudy ascitic fluid in pelvic cavity, right iliac fossa, and the liver surface. It's almost normal in 
uterus, left fallopian tube, bilateral ovaries roughly, the right fallopian tube was absent. there was no apparent lesion in the surface of omentum majus, appendix, liver and diaphragm. After careful observation, a friable tumor measuring $1.0 \times 0.8 \times 0.5 \mathrm{~cm}$ was discovered at the fimbriae of the left fallopian tube. Laparoscopic hysterectomy, left salpingo-oophorectomy and right oophorectomy was performed. Frozen section of the tumor at the distal part of the left fallopian tube revealed serous carcinoma. The opposite fallopian tube, uterus, and both ovaries were normal. Given the circumstance, according to the principles of treatment, omentectomy, appendicectomy, pelviclymphadenectomy and paraortic lymphadenectomy were be continue to be done. Microscopic examination of the formalin-fixed and paraffin-embedded surgical specimens confirmed the diagnosis of high-grade serous Carcinoma of Fallopian Tube of the fimbriae of the left fallopian tube, with local invasion of deep tube wall and one righ pelvic lymphnode metastases. The remainder of the surgical material were without cancer cell. Immunohistochemical staining was positive for P53, WT-1, P16, CK7, CK20, Calretinin , and slightly positive for ER, Ki-67(almost 70\%). Final diagnosis was Primary high-grade serous carcinoma of fallopian tube, with extension to the pelvic lymphnode and metastases to the umbilicus. Therefore, according to FIGO surgical-pathological stage, this case was in stage $\mathbb{}$ C.

On the sixth day after operate, reexamination serum tumor markers levels were: CA 125: $68.02 \mathrm{U} / \mathrm{ml}, \mathrm{CA}$ 19 - 9: 55. 96U/ml. Next day, she received the standard combination chemotherapy including six cycles of paclitaxel $175 \mathrm{mg} / \mathrm{m}^{2}$ and carboplatin $(A \cup C=5)$ at 3- weeks intervals. Combined with patient's height and weight, she got paclitaxel $270 \mathrm{mg}$, and carboplatin $500 \mathrm{mg}$ every time. During chemotherapy, the patient showed no signs of a non-response. Her postoperative course was begnign and was discharged on June, 2020. Several days later, the gene detection demonstrated BRCA1 frameshift mutation. On the twentieth day after the first chemotherapy, reexamination serum tumor markers levels were: CA 125: 25. $85 \mathrm{U} / \mathrm{ml}, \mathrm{HE}$ 4: $70.47 \mathrm{pmol} / \mathrm{l}$. Thus, treatment with the former. Last reexamination serum tumor markers levels were: CA 125: $12.79 \mathrm{U} / \mathrm{ml}$, To date, 2 days after six cycles chemotherapy. there is no obvious side effect. The patient is alive without recurrence.

\section{Discussion}

The current report of the patient with PFTC deserves well sharing. Our aim was to execute personalized dignosis and treatment to reduce the incidence rate and prolong life cycle. PFTC was first reported by Rokitansky in $1847^{5}$. The incidence rate of primary fallopian tube carcinoma accounts for $0.14 \%-1.8 \%$ of all gynecological malignancies ${ }^{6}$ However, recent data suggest that the true incidence of PFTC has been substantially underestimated. A large number of evidence has confirmed that most epithelial ovarian cancers(EOC)are of fallopian origin. And it originates from the epithelium of the fallopian tube. The most common histological type of it is serous adenocarcinoma ${ }^{7}$. Postmenopausal women are susceptible. Although nosogenesisis unclear, a lot of datas suggest genetic factor as unanimous principal factor.

The routes of metastasis of fallopian tube carcinoma are similar to those of ovrican cancer. PFTC mainly spreads to adjacent tissue or organ, and also can disseminate to other sites by lymphatic or 
hematogenous routes. Lymphatic metastasis of fallopian tube carcinoma has three main pathways ${ }^{8}$. PFTC spreads mainly through the infundibulopelvic ligament to the paraaortic lymph nodes, and also through broad ligament to the pelvic lymph nodes and through round ligament to the inguinal lymph nodes ${ }^{4}$. Umbilical metastasis from intraabdominal cancer mainly through the lymphatic channels draining the paraaortic lymph nodes, but may also spread through direct extension, venous routes, and embry-onic remnants ${ }^{9}$. Sister Mary Joseph $(1856$ - 1939) was the first to notice that an umbilical nodule may be a specific sign of intraabdominal cancer. It has consequently been suggested that the name "Sister Mary Joseph's nodule" should be given to metastatic tumors of the umbilicus ${ }^{4}$ Because of the fist sign in umbilical region, as well as its clinical feature same with omphalitis, logically, she was dignosed as omphalitis at first. From her postoperative pathology result, we overturned the conclusion.

Hereditary tumor syndrome is a disease-causing mutation of one or more genes that causes tumors in one or more organs of an individual, and the mutated genes can be inherited from generation to generation in the family line, most of which are autosomal dominant, accounting for about $5 \% \sim 10 \%$ of all tumors ${ }^{10}$. Hereditary tumors are associated with germline mutations in oncogenes or tumor suppressor genes in the carcinogenesis pathway. Hereditary breast and ovarian cancer(HBOC) as one of the most genetically related syndromes in ovarian cancer is related to the pathogenic mutation of tumor suppressor genes. The majority HBOC are mutation of BRCA 1 and BRCA2. The NCCN Guidelines recommended, all women diagnosed with ovarian, fallopian tube, or abdominal cancer, regardless of age or family history, should receive inheritance counseling and undergo BRCA genetic testing ${ }^{11}$. BRCA (Breast Cancer Susceptibility Genes) includes BRCA1 and BRCA2. There are hundreds of BRCA1/2 mutations. These two genes are responsible for encoding and synthesizing a tumor suppressor protein, which participates in the repair of DNA homologous damage and prevents excessive cell proliferation. This protein and Poly ADP -ribose polymerase(PARP)serve as two ways to repair DNA damage, When one of the repair pathways fails, the damaged part of the DNA can be repaired through another pathway to stabilize the genetic information in the cell. When a cell has a BRCA1/2 mutation, although it loses the BRCA repair pathway, it can also repair DNA damage through the PARP pathway, so the mutated tumor cells can still survive. At present, the targeted drugs for BRCA are mainly PARP inhibitors, which block the survival of BRCA-mutated tumor cells by inhibiting the PARP pathway, resulting in the death of tumor cells. The mutation has also been linked to a higher risk of pancreatic and prostate cancers.

Through genetic testing, Carriers of tumor-causing genes should be timely screened for cancer. Screening methods are commonly used in clinical practice, such as gynecological ultrasound, CA125 detection. latrogenic intervention can effectively reduce the risk of hereditary gynecological tumors, including drug prophylaxis and risk reduction surgery. Preventive drugs are mostly sex hormone drugs, mainly oral contraceptives. Preventive bilateral salpingo-oophorectomy, bilateral salpingectomy, and hysterectomy can reduce the risk of cancer in carriers of hereditary gynecological tumor mutation genes, so it is called risk reduction surgery. Patients with hereditary tumors are relatively young at the time of onset, and it is recommended that high-risk patients undergo risk-reduction surgery at the age of 35 to 45 or after the completion of childbirth. Iatrogenic menopause, infertility, and hypoestrogen syndrome caused by risk- 
reducing surgery can be intervened. In combination with the patient, the mother, sister, brother and son of the first-degree relatives should be genetically tested. Prophylactic removal of the ovaries can reduce the incidence of breast cancer. The cost of genetic testing is not high, all should be implemente. Prevention is based on the results of the examination. Preventive resection of the prostate is not recommended. Regular PSA,digital rectal examination, and transrectal ultrasound examination, should be routine screening once a year from $40-45$ years old.

The ideal balance between tumor prevention and fertility preservation in genetic gynecological tumor population is risk-reducing surgery after fertility completion. The optimal age for RRSO implementation is 35 to 45 years old. The "childbearing period" time window is relatively shorter for women at high risk of gynecological tumors. If they have not completed their childbearing and have to consider receiving RRSO risk reduction surgery, it is recommended to use frozen egg or frozen embryo assisted reproductive techniques (ART) to complete the childbearing. Carriers of B R C A 1 and B R C A 2 mutations are diagnosed as gynecological tumors. Fertility preservation (FP) also needs to take into account chemotherapy-related infertility, premature ovarian insufficiency (POI), or iatrogenic menopause, etc. Gynecological oncologists must have the awareness and concept of oncofertility, and guide B R C A 1 and B R C A 2 mutation carriers to go to the department of reproductive endocrinology, so as to have relatively ample time to assess basic ovarian reserve, guide and implement fertility preservation plans. Patients can also fully understand the timing of reproductive function preservation and the pros and cons of pre-implantation genetic testing. RRSO recipients who have preserved frozen eggs or embryos can achieve fertility by in vitro fertilization and embryo transfer(IVF-ET). Otherwise, donating eggs can be considered. Adoption is also an option. Uterine transplantation is a new technique to realize family planning. Recently, a case of live birth was reported. However, for women with high risk of hereditary gynecological tumors, the role and risk of uterine transplantation in preserving fertility have not been determined. Hereditary gynecological tumors are inherited in an autosomal dominant manner, and carriers of the mutant gene have a $50 \%$ probability of passing the pathogenic mutation to their offspring ${ }^{12}$. PGD/PGS and prenatal diagnosis techniques can minimize the risk of the transmission of pathogenic mutations to offspring. Women with pathogenic gene mutations are at higher risk of cancer. Such patients should receive genetic counseling related to PGD/PGS. PGD/PGS is first obtained through in vitro fertilization, the embryos (often at the blastocyst stage) are biopsied, and genetic testing is used to screen embryos for transplantation that do not carry pathogenic genetic mutations. When PGD/PGS for monogenic/single gene defects is done, PGD/PGS for aneuploidy can be tested meanwhile. Different from prenatal diagnosis, PGD/PGS can identify embryos carrying pathogenic mutation genes before pregnancy, effectively avoiding worries and considerations during pregnancy, and avoiding termination of pregnancy when pathogenic mutation is found in embryos. It is recommended that carriers with BRCA $1 / 2$ mutations undergo transvaginal ultrasound combined with serum CA125 screening from 30 to 35 years of age. The methods with early diagnosis technology still need to be developed.

With the development of society, the world pays more attention to health, The world is looking for a code to prevent disease. Medical technology is advancing so fast that it can solve many of the technical 
problems of the past and bring good news to people. Standardized consultation for hereditary tumors is helpful for screening and identifying high-risk individuals, and guide intervention measures based on genetic principles. It is not only beneficial to the prevention and early detection of tumors, but also can be involved in the selection of fertility decisions and subsequent endocrine therapy. Therefore, Gynecologic oncologists should cooperate with reproductive medicine doctors to provide fertility preservation, PGD/PGS and other related consultations for high-risk patients with gynecological tumors, and formulate diagnosis and treatment plans. With the development of gene detection technology, effective intervention measures should be implemented. It is very important to realize early prevention, early diagnosis and early treatment for cancer patients with familial genetic tendency.

\section{Declarations}

\section{Funding}

This research was supported by grants from the National Natural Science Foundation of China (NO. 81301647).

\section{Conflicts of interest}

The authors declare that there is no conflict of interests.

\section{Ethics approval}

Not applicable

\section{Consent to participate}

Written informed consent was obtained from individual or guardian participants

\section{Consent for publication}

Not applicable

\section{Availability of data and material}

All data generated or analysed during this study are included in this published article

\section{Code availability}

Not applicable

\section{Authors' contributions}

$\mathrm{XC}$ and NZ made contributions to the acquisition of the case. XC made contribution to the writing of the case report. XC and GW have downloaded data and documents. YF, MZ, and YL processed the charts and 
formato. All authors made an equal contribution to this work and reached an agreement on the final manuscript version.

\section{References}

[1] Jonathan S. Berek, Sean T. Kehoe, Lalit Kumar, et al. Cancer of the ovary, fallopian tube, and peritoneum. Int J Gynaecol Obstet. 2018, 143: 59-78.

[2] Couturier M G, Neale R C. Adenocarcinoma of umbilicus secondary to Fallopian tube carcinoma.. Am J Obstet Gynecol 1969, 105(1): 32 - 4.

[3] Konstantinopoulos Panagiotis A, Norquist Barbara, Lacchetti Christina, et al. Germline and Somatic Tumor Testing in Epithelial Ovarian Cancer: ASCO Guideline.. J Clin Oncol 2020, 38(11): 1222-1245.

[4] Boris Kirshtein, Mihai Meirovitz, Elimelech Okon, et al. Sister Mary Joseph's nodule as the first presenting sign of primary fallopian tube adenocarcinoma. J Minim Invasive Gynecol 2006, 13(3): 234236.

[5] Sun Minming, Bao Lingjie, Yi Xiaofang, et al. Retrospective analysis of clinical characteristics and prognostic factors in 101 patients with primary fallopian tube carcinoma. Chin Oncol, 2019, 29(09): 723729.

[6] Jiang Jian, Chen Zhi-Peng, Zhu Hui-Ping, et al. Responses of metastatic primary fallopian tube carcinoma to pembrolizumab and nab-paclitaxel: A case report.. Medicine 2020, 99(28): e21203.

[7] Gungorduk Kemal, Ertas Ibrahim E, Ozdemir Aykut, et al. Prognostic Significance of Retroperitoneal Lymphadenectomy, Preoperative Neutrophil Lymphocyte Ratio and Platelet Lymphocyte Ratio in Primary Fallopian Tube Carcinoma: A Multicenter Study.. Cancer Res Treat 2015, 47(3): 480-8.

[8] Xie Zhanling, Yao Guang, Qu Bo, et al.. A case of primary fallopian tube carcinoma with extensive retroperitoneal metastasis in early stage. Chin J Lab Diagn, 2017, 21(02): 336-337.

[9] Dubreuil A, Dompmartin A, Barjot P, Louvet S, Leroy D. Umbilical metastasis or Sister Mary Joseph's nodule. Int J Dermatol. 1998; 37: 7-13.

[10] Hereditary Cancer Syndromes and Risk Assessment: ACOG COMMITTEE OPINION, Number 793.. Obstet Gynecol 2019, 134(6): e143-e149.

[11] Chang Xiaohong, Li Yi, Cui Heng. Clinical significance of genetic counseling for ovarian cancer[J]. Chin J Clin Obstetrics GynEcol, 2020, 21(05): 449-451.

[12] Wang Yudong, Wang Yingmei, Wang Jiandong, et al. Expert Consensus on management of high-risk population of hereditary gynecological tumors (2020). Chin J Pract Gynecol Obstet, 2020(9). 825-834. 


\section{Tables}

Table 1. BRCA1/2 GENETIC TESTING

\begin{tabular}{|ll|}
\hline TEST ITEM \\
\hline DX0513 BRCA1/2 GENETIC TESTING \\
\hline TEST RESULT \\
\hline Disease & Hereditary breast cancer/Hereditary ovarian cancer \\
\hline Gene(NM) & BRCA1(NM_007294. 3) \\
\hline Nucleotide changes & c. 1961dupA \\
\hline Amino acid changes & p. Tyr655Valfs*18 \\
\hline Gene subregion & CDS9 \\
\hline Heterozygosity & Het \\
\hline Function change & Frameshift \\
\hline Genetic method & AD \\
\hline Mutation type & Known pathogenic mutations \\
\hline Het: Heterozygous mutation AD: Autosomal dominant inheritance \\
\hline
\end{tabular}

\section{Figures}



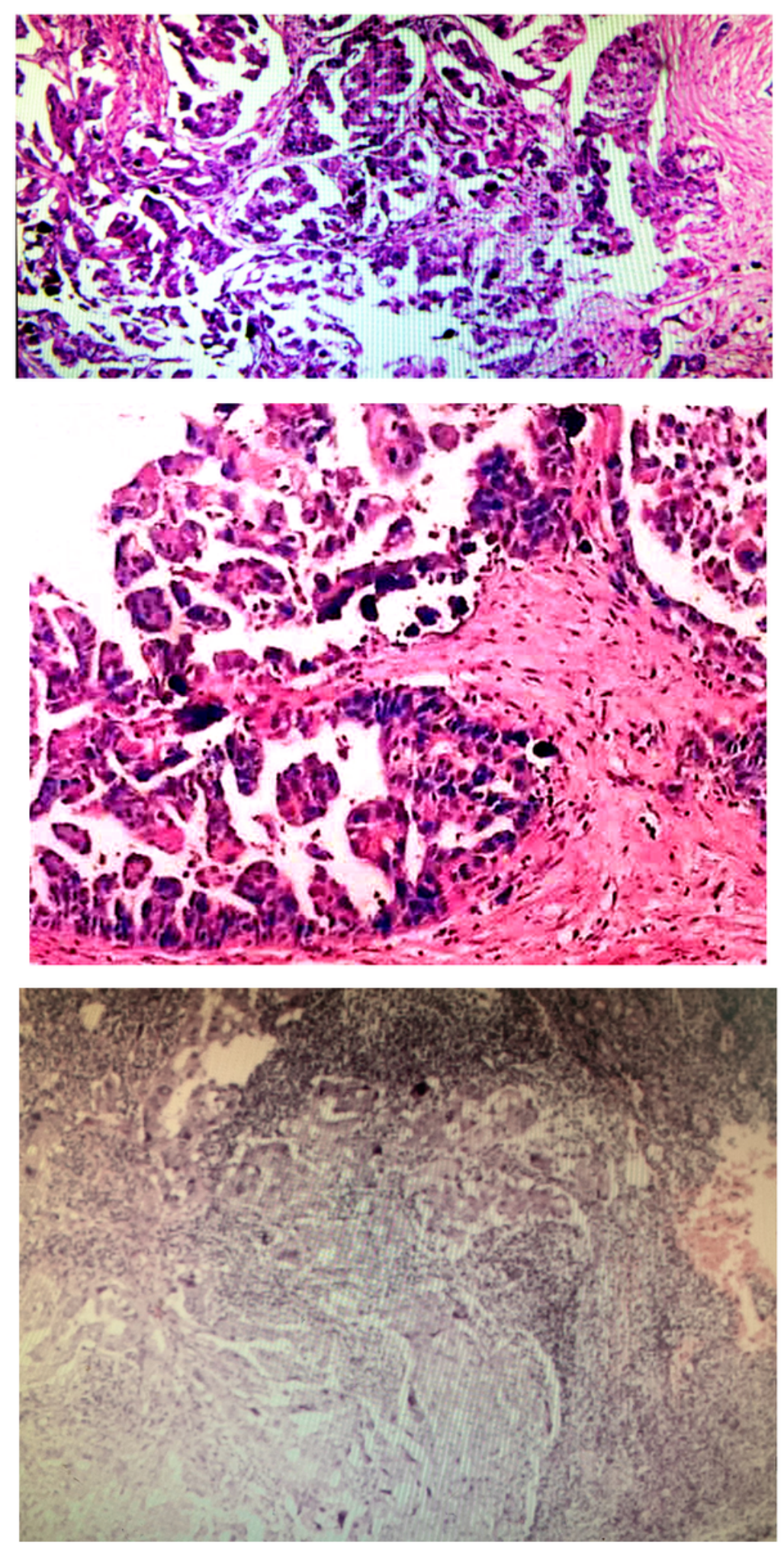

Figure 1

Microscopic examination of the formalin-fixed and paraffin-embedded surgical specimens confirmed the diagnosis of high-grade serous Carcinoma of Fallopian Tube of the fimbriae of the left fallopian tube, with local invasion of deep tube wall and one righ pelvic lymphnode metastases. The remainder of the surgical material were without cancer cell. Immunohistochemical staining was positive for P53, WT-1, P16, CK7, CK20, Calretinin \and slightly positive for ER, Ki-67(almost 70\%) 